
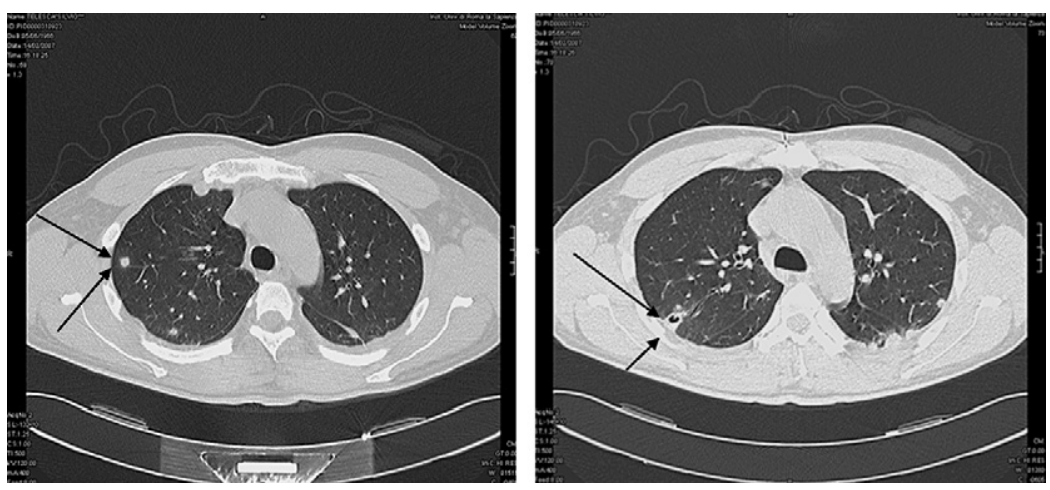

FIGURE 1. Preoperative computed tomography scan showing a pulmonary infiltrate mimicking pneumonia, which is likely secondary to septic emboli.

TABLE 1. Key elements for timing of surgery in patients with rightsided infective endocarditis

- Infecting organism (esp. Staphylococcus aureus and Gram-negative bacilli)

- Secondary heart failure

- Severity of valvular dysfunction

- Response to appropriate antibiotic therapy

- Presence of abscesses, fistulae, pseudoaneurysms, or local extension

- Pulmonary septic emboli, abscesses, or cavitations

- Systemic involvement (DIC, acute renal failure)

$D I C$, Disseminated intravascular coagulopathy.

patient, it is preferable to avoid any unnecessary prolongation of the procedure. Finally, a pulmonary homograft is used to produce least turbulence, and these valves are considered the best substitute for the pulmonary valves.

\section{CONCLUSIONS}

Despite the remarkable resistance of pulmonary homografts to infection in the context of the Ross procedure, this case illustrates the importance of meticulous echocardiographic examination of all valves to eliminate endocarditis. Pulmonary valve endocarditis often presents insidiously and mimics pulmonary infections, therefore warranting a high degree of suspicion. Finally, timing of surgical intervention is critical for the patient's overall prognosis and for protection of the unaffected pulmonary autograft.

\section{References}

1. Yacoub MH, Klieverik LM, Melina G, Edwards SE, Sarathchandra P, Bogers AJ, et al. An evaluation of the Ross operation in adults. J Heart Valve Dis. 2006;15: 531-9.

2. Kang N, Smith W, Greaves S, Haydock D. Pulmonary valve endocarditis. N Engl J Med. 2007;356:2224-5.

3. Zeledon JI, McKelvey RL, Servilla KS, Hofinger D, Konstantinov KN, Kellie S, et al. Glomerulonephritis causing acute renal failure during the course of bacterial infections. Histological varieties, potential pathogenetic pathways and treatment. Int Urol Nephrol. 2008;40:461-70.

4. Bonow RO, Carabello BA, Chatterjee K, de Leon AC Jr, Faxon DP, Freed MD, et al. 2008 Focused update incorporated into the ACC/AHA 2006 guidelines for the management of patients with valvular heart disease. Circulation. 2008;118: e523-661.

\title{
Subepicardial congenital vascular malformation mimicking a bulky tumor with severe compression of the right heart chambers
}

\author{
Naser Qedra, MD, ${ }^{\mathrm{a}}$ Takeshi Komoda, MD, ${ }^{\mathrm{a}}$ Marian Kukucka, MD, ${ }^{\mathrm{b}}$ and Roland Hetzer, MD, PhD, ${ }^{\mathrm{a}}$ Berlin, \\ Germany
}

\footnotetext{
From the Departments of Cardiothoracic and Vascular Surgery ${ }^{\mathrm{a}}$ and Anesthesiology, ${ }^{\mathrm{b}}$ Deutsches Herzzentrum Berlin, Berlin, Germany.

Disclosures: None.

Received for publication Feb 13, 2009; accepted for publication Feb 22, 2009; available ahead of print May 1, 2009.

Address for reprints: Naser Qedra, MD, Deutsches Herzzentrum Berlin, Augustenburger Platz 1, 13353 Berlin, Germany (E-mail: qedra@gmx.de).

J Thorac Cardiovasc Surg 2010;139:e100-2

$0022-5223 / \$ 36.00$

Copyright (c) 2010 by The American Association for Thoracic Surgery

doi:10.1016/j.jtcvs.2009.02.039
}

A 52-year-old man was admitted in poor general condition to the emergency department owing to increasing dyspnea and swelling in both legs over 4 weeks. In addition, he had lost about $8 \mathrm{~kg}$ in weight during the past 2 months. The electrocardiogram showed no pathologic findings but left axis deviation. The chest radiograph showed no abnormalities. Transthoracic echocardiography showed an inhomogeneous structure compressing the right atrium (RA) and the right ventricle $(\mathrm{RV})$. The transesophageal echocardiogram showed 

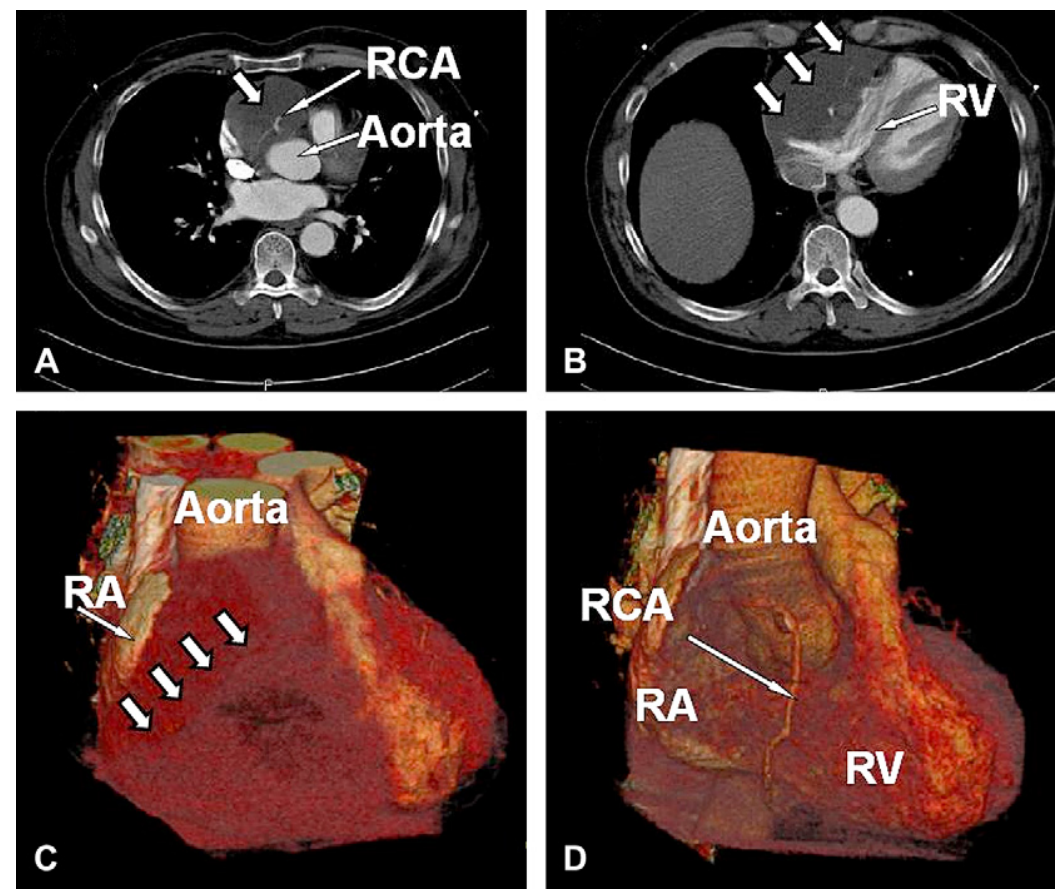

FIGURE 1. A and B, Contrast-enhanced electrocardiogram-gated multislice spiral computed tomograms. C and D, Color-coded, semitransparent, 3-dimensional reconstruction in angulated coronal planes and color-coded endoluminal image. $R A$, Right atrium; $R V$, right ventricle; $R C A$, right coronary artery. Short arrows show malformation mass.

a bulky inhomogeneous mass including lacunae located parallel to the right side of the heart with severe compression of the RA as well as the RV. No incompetence of the tricuspid valve was observed. The left ventricular ejection fraction was $70 \%$ and the end-diastolic diameter $52 \mathrm{~mm}$.
Contrast-enhanced electrocardiogram-gated multislice spiral computed tomographic scan depicted a well-defined intrapericardial mass measuring $5 \times 10 \mathrm{~cm}$ anterior to the ascending aorta, RA, and RV and compressing both cavities. It extended posteriorly along the atrioventricular sulcus and
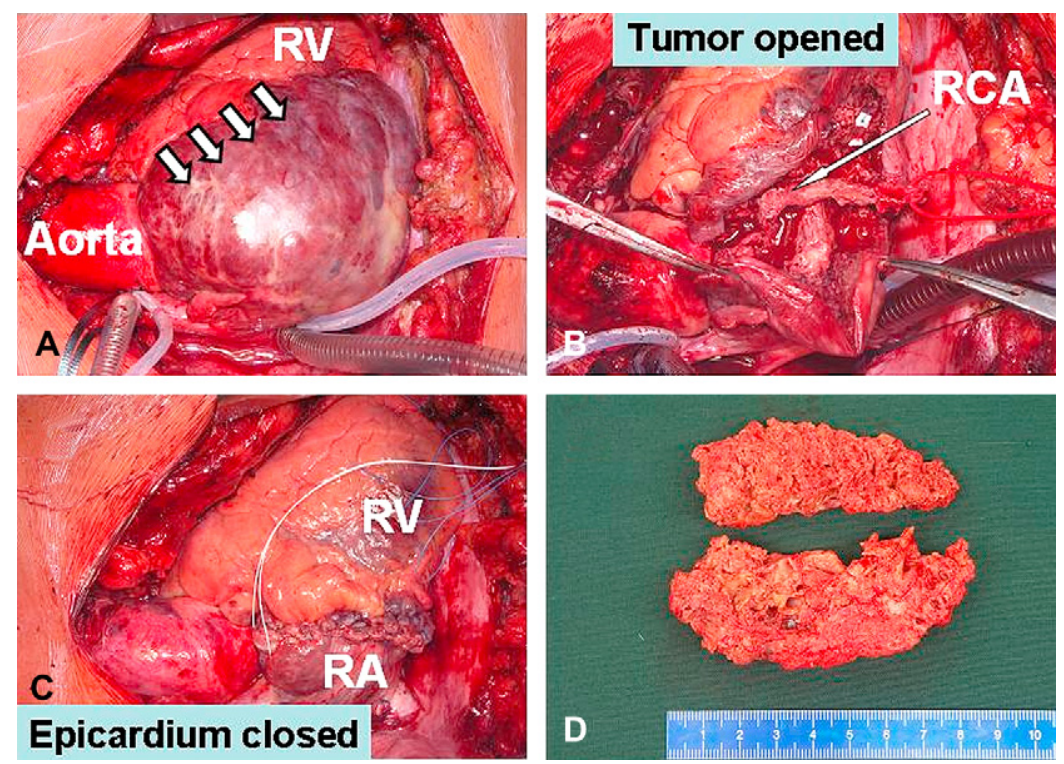

FIGURE 2. Intraoperative images: A, after opening of the pericardium; B, after opening of the mass; $\mathrm{C}$, after tumor mass resection; D, showing the resected mass. $R A$, Right atrium; $R V$, right ventricle; $R C A$, right coronary artery. 
surrounded the right coronary artery (RCA), which supplied the tumor. Color-coded, semitransparent, 3-dimensional reconstruction in angulated coronal planes and color-coded endoluminal view demonstrated the extension of the tumor along the atrioventricular sulcus and surrounding the RCA (Figure 1). In the coronary angiogram, coronary artery disease was excluded. The RCA was without pathologic findings but had branches supplying the tumor.

The patient underwent a median sternotomy. There was a bulky livid-colored mass extending from the ascending aorta, anterior to the RA and the RV, down to the acute margin of the heart. On cardiopulmonary bypass and without crossclamping of the aorta, the tumor was opened and the RCA was seen to be encased by the mass from the origin to the crux. The branches originating from the RCA and supplying the tumor were divided and clipped. The tumor was radically removed and the epicardium closed (Figure 2). The postoperative course was uneventful and currently the 9-month follow-up is event free. The histologic diagnosis of benign, congenital vascular malformation with hematic and lymphatic involvement was made by immunohistochemical staining, which was positive for muscle actin, endothelial cells (CD31), and lymphatic cells (D2-40).

\section{DISCUSSION}

Congenital vascular malformations are developmental aberrations of hematic and/or lymphatic vessels ${ }^{1,2}$ and occur in approximately $0.3 \%$ to $0.5 \%$ of the population. ${ }^{3}$ They are characterized by flat endothelium, and growth of the lesion is commensurate with growth of the individual. On the basis of anatomic location, congenital lymphatic malformations have also been described: $75 \%$ are located in the neck, $20 \%$ in the axillae, $2 \%$ are intra-abdominal/retroperitoneal, $2 \%$ are in limbs and bones, and $1 \%$ are in the mediastinum. ${ }^{4}$ However, to our knowledge, involvement of the heart has not been described before.

This is the first case of an adult patient with a bulky subepicardial congenital vascular malformation surrounding the
RCA, supplied by it, and causing severe compression of the right heart chambers. The absence of cardiac symptoms over the years suggests the long-term growth of the malformation. Initially, because of the subacute onset of the cardiac symptoms accompanied by loss of weight and because of the inhomogeneous character in echocardiograms and computed tomographic scans, a malignant mediastinal process was presumed. The expansive and compressive growth of the mass complies with the described behavior of vascular malformations with lymphatic involvement. ${ }^{1}$ The loss of weight can be interpreted as cardiac cachexia. The late onset of the cardiac symptoms may be explained by the ability of the heart to compensate compression over time, until a critical point is reached. Owing to the use of advanced diagnostic procedures, it was possible not only to radically resect the tumor but also to save the RCA.

In conclusion, this report demonstrates that congenital vascular malformations may manifest also in the heart. As such malformations with lymphatic involvement show expansive and compressive growth, continuous monitoring is recommended in asymptomatic patients and surgical removal should be considered as the therapy of choice. In this condition, a multidisciplinary approach, including advanced diagnostics, is necessary to achieve optimal results.

We thank A. Benhennour for literature research, A. Gale for editorial assistance, and Natalia Solowjowa and Rainer Roettgen for editing the computed tomographic scan.

\section{References}

1. Cohen MM Jr. Vascular update: morphogenesis, tumors, malformations, and molecular dimensions. Am J Med Genet. 2006;140A:13-2038.

2. Connell F, Homfray T, Thilaganathan B, Bhide A, Jeffrey I, Hutt R, et al. Congenital vascular malformations: a series of five prenatally diagnosed cases. Am J Med Genet. 2008;146A:2673-80.

3. Mulliken JB, Virnelli-Grevelink S. Vascular anomalies. In: Freedberg I, ed. Fitzpatrick's dermatology in general medicine. New York: McGraw-Hill; 1999:1175-94.

4. Anderson NG, Kennedy JC. Prognosis in fetal cystic hygroma. Aust N Z J Obstet Gynaecol. 1992;32:36-9. 